\title{
Thermal Stability of Domains in Magneto-Optic Recording Materials
}

\author{
Mark H. Kryder \\ Data Storage Systems Center Carnegie Mellon University, Pittsburgh, PA 15213-3890, USA
}

The thermal stability of magnetic recordings has recently become a topic of considerable interest, because modeling has shown ${ }^{1}$ that, under the assumption of linear scaling, longitudinal magnetic recording in fine grain polycrystalline media will encounter thermal instabilities at a density of $36 \mathrm{Gbit} / \mathrm{in}^{2}$. Data have also been collected, which indicate that fine-grain magnetic recording media indeed do exhibit significant magnetization decay and these data support the predictions. Theoretical modeling indicates that, if the bit cell in magnetic recording is made more square than the typical $15: 1$ or $20: 1$, which is common today, the density at which magnetic recordings in longitudinal media become thermally unstable may be increased to the order of $100 \mathrm{Gbit} / \mathrm{in}^{2}$. Nevertheless, means remain to be found to achieve stable magnetic recordings at densities beyond $100 \mathrm{Gbit} / \mathrm{in}^{2}$.

In the work described here, the thermal stability of magnetic domains in Co/Pt multilayer magnetic materials was determined by measuring the thermal stability of domain walls and the thermal decay of magnetization and coercivity under the influence of applied fields. The decay of normalized coercivity with time for three different films having different coercivities is plotted in Fig. 1, where it is seen that, even for the film with a coercivity of only $560 \mathrm{Oe}$, the coercivity does not decay to zero for times shorter than $10^{15} \mathrm{secs}$. From data such as these it was found that the activation energy for wall motion in these materials was in the range of $60 \mathrm{~K}_{\mathrm{B}} \mathrm{T}$ to $140 \mathrm{~K} \mathrm{~B}$, implying a lifetime of over 30 million years in an applied field of $100 \mathrm{Oe}$.

To account for the effects of domain wall surface tension, which tends to cause very small domains to collapse, an effective field, which accounts for the pressures from domain wall surface tension and demagnetizing fields, was introduced. This effective field model can then be used to predict the thermal stability of domain walls with very small radii of curvature. Based upon this analysis, it is projected that circular domains in a Co/Pt multilayer film having a coercivity of about $1760 \mathrm{Oe}$, would have a lifetime against thermal decay in excess of 10 years.

Empirically, it was also noted that the thermal activation energy in the films examined increases approximately linearly with the product $\mathrm{M}_{s} \bullet \mathrm{H}_{\mathrm{c}}$, where $\mathrm{M}_{\mathrm{s}}$ is the saturation magnetization of the film and $\mathrm{H}_{\mathrm{c}}$ is the measured coercivity. This suggests that, if $\mathrm{Co} / \mathrm{Pt}$ multilayer films can be made with higher values of $\mathrm{M}_{\mathrm{s}} \bullet \mathrm{H}_{\mathrm{c}}$, then even smaller domains would be stable.

A domain size of $20 \mathrm{~nm}$ implies a potential square bit cell of $40 \mathrm{~nm}$. This corresponds to an areal density of 400 Gbit/in ${ }^{2}$, using pulse position modulation and could support densities in excess of $800 \mathrm{Gbit} / \mathrm{in}^{2}$ with pulse width modulation, if adequate signal to noise ratio could be achieved.

Reference:

1. S.H. Charap, Pu-Ling Lu and Yanjun He, IEEE Trans. Magnet., 33, 978 (1997). 


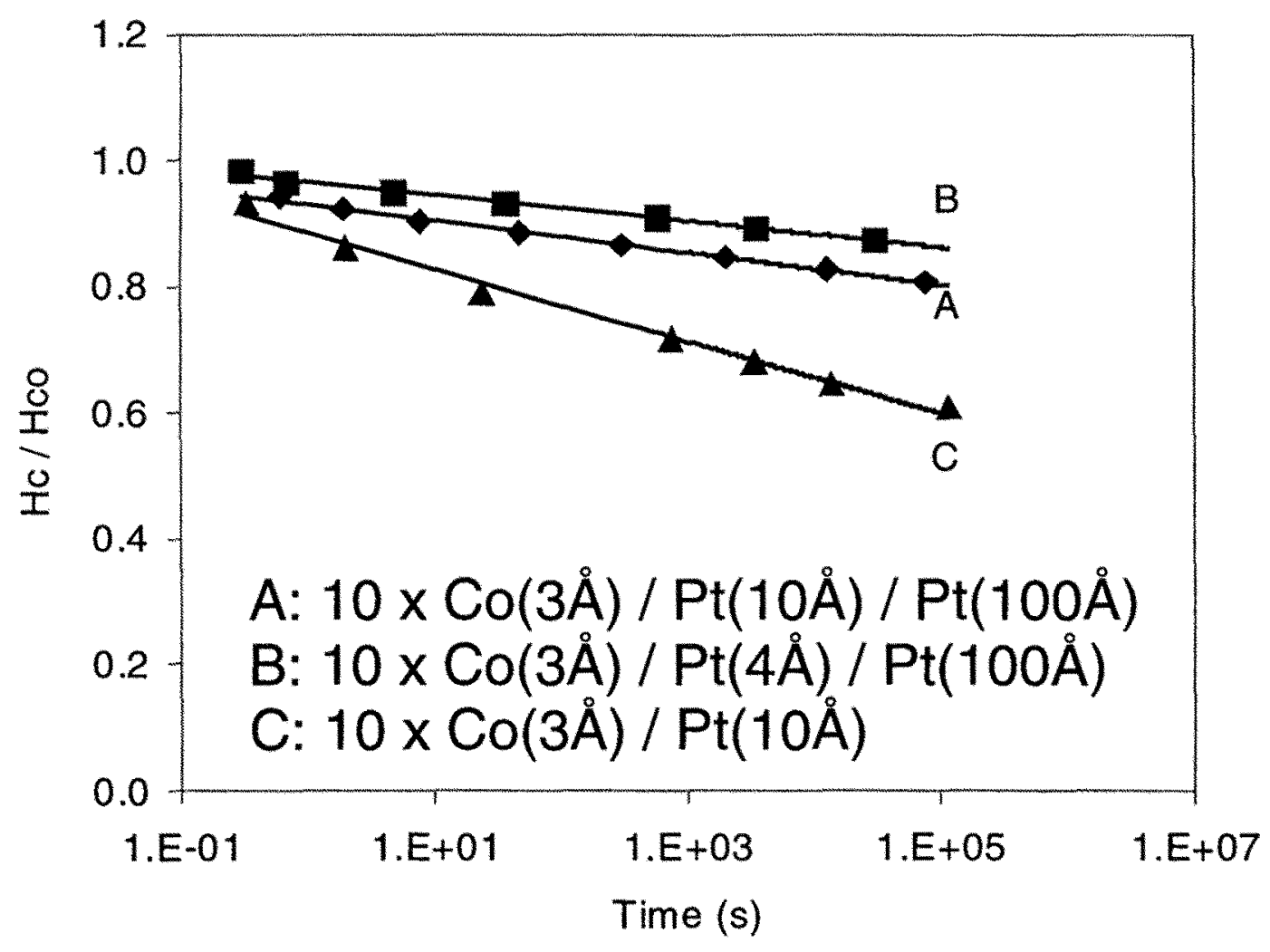

Fig. 1 A plot of normalized coercivity as a function of time for three different Co/Pt multilayer films having coercivities of A) $1040 \mathrm{Oe}, \mathrm{B}) 1760 \mathrm{Oe}$ and C) $560 \mathrm{Oe}$. 